\title{
Design and Implementation of a Patchouli Growth Environment Monitoring System Based on IoT
}

\author{
Guodong Li ${ }^{1, *}$ and Ping Zhang ${ }^{2, *}$ \\ ${ }^{1}$ College of Informatics, Huazhong Agricultural University, Wuhan, Hubei 430070, China \\ ${ }^{2}$ School of Computer, BaoJi University of Arts and Sciences, Baoji 721016, China \\ * Corresponding author: Guodong Li (AGuodong_Li@163.com), Ping Zhang (jcjyxy@163.com)
}

Manuscript submitted 24 May 2021; Accepted 26 May 2021; Published 28 May 2021

Academic Editor: Jinchao Chen

Abstract: With the development of the times, the concept of 'interconnection of all things' has been deeply rooted in the hearts of people. As an early and developing Internet technology, Internet of things (IoT) has shown its infinite potential in many fields of social life. In agriculture, the agricultural IoT is covering a growing number of fields including greenhouse planting, climate factor collection, climate prediction and so on. So that idea of patchouli growth environment monitoring system is put forward. In this paper, the medicinal plant "patchouli" is studied, and its medicinal value and growth factors are discussed. Meanwhile, based on ZigBee technology, we design a set of feasible monitoring scheme including hardware and software. The hardware part used ZigBee wireless network design, mainly relies on the cc2530 chip and the esp8266 wifi chip to carry on the wireless distance transmission to the sensor data. It interacts between OneNET cloud server and mysql database through MQTT protocol, TCP/IP protocol and HTTP protocol.

Index Terms: Medicinal plants, Patchouli, IoT, ZigBee.

\section{Introduction}

China is one of the countries with the most abundant medicinal plant resources. It has a long history of the discovery, use and cultivation of medicinal plants. Ancient Chinese historical materials shows that the discovery and use of medicinal plants is the result of the gradual accumulation of experience and knowledge of ancient humans through their long - term life and production practice. As a kind of medicinal plant, patchouli has considerable commercial value and cultivation value. In the past, the cultivation of patchouli required a large number of artificial participation in the cultivation, and could not achieve real-time and efficient monitoring. With the development of science and technology, agriculture and planting have been changing from traditional to modern planting. Modern planting industry no longer relies on labor to improve industrial efficiency, but starts from the systematic and automated management direction to improve its industrial efficiency.

Modern planting is actually designed with hardware and software, that is, by laying the corresponding sensor equipment in the farmland and other plantation areas, the temperature, humidity, light, wind, rainfall, soil humidity and other environmental elements are recognized and collected. Then people realize information data transmission through 
wireless sensing network, cloud server and database. Through real-time and diverse agricultural data, people can realize the efficient development of planting industry.

Research on environmental monitoring has never stopped. The US first invented computers and to use computers for greenhouse control and management. Its developed planting technology and planting capacity also created conditions for the development of its environmental control technology. Facilities gardening technology in the Netherlands is currently widely recognized globally and its greenhouse environmental control taking into account the plant growth cycle and six major factors affecting plant growth [1]. Israel's natural environment is mainly plains, hills, rift valleys and desert. On this basis, Israeli scientists have developed special plastic films that allow light, climate control, antiviral, UV protection and capable of natural corrosion and built a modern desert greenhouses and greenhouses [2]. The Netherlands, Belgium have early used $\mathrm{C} / \mathrm{S}$ mode to use specific software to monitor and control the operation of relevant equipment on site, with the technical basis of network and wireless communication. Since the $1960 \mathrm{~s}$, Japan's modern greenhouse agriculture has entered a stage of rapid development. Its core, in addition to the transformation of the greenhouse itself (the greenhouse style gradually changes from loose structure and small scale), is automation [3]. The UK also attaches great importance to the monitoring and control of environmental parameters such as biological growth and temperature and humidity. They are good in greenhouse computer remote sensing technology and can control the greenhouse environmental parameters, with a long distance of more than 50 kilometers.

In fact, because the intelligent greenhouse control system is expensive and requires a lot of financial input in the actual use process, the users of the intelligent greenhouse control system are mainly some large enterprises and companies. We try to achieve patchouli growth environment monitoring in a simple and low way.

\section{Cultivation conditions of patchouli}

Patchouli is preferred in areas with an annual average temperature of $19 \sim 26{ }^{\circ} \mathrm{C}$, growing slowly or stopping at temperatures above $35{ }^{\circ} \mathrm{C}$ or below $16{ }^{\circ} \mathrm{C}$. It is suitable for growing in a wet, rainy environment, afraid of drought, and requires an annual rainfall of more than 1, $600 \mathrm{~mm}$. Patchouli seedling period like rain, and like the humidity environment in the long term (but the soil humidity is too high, it will also die.) [4]. Irrigation should be noted in areas with less rainfall. Patchouli maturity can grow under full light, but shed should be built to cover the sun during Patchouli seedlings. Its root is more cold resistant, and can overwinter in the north.

Patchouli has not strict requirements for soil, but it prefers a fertile and loose sandy loam. The life of Patchouli seed is 2 to 4 years, and the seed germination needs light conditions. Seed germination suitable temperature is between $18{ }^{\circ} \mathrm{C}$ and $22{ }^{\circ} \mathrm{C}$. Therefore, the monitoring system generally mainly collects the growth of the temperature, humidity, soil temperature, humidity and illumination of the surrounding environment.

\section{3. system design}

In hardware design, the equipment layout is mainly determined according to the actual environment, while the sensor data is transmitted according to ZigBee component (wifi module, etc.). In software design, the corresponding reception interface is designed according to various environmental parameters, obtaining historical data according to the data of the cloud platform and stored into the database. Technical route is shown in Figure 1.

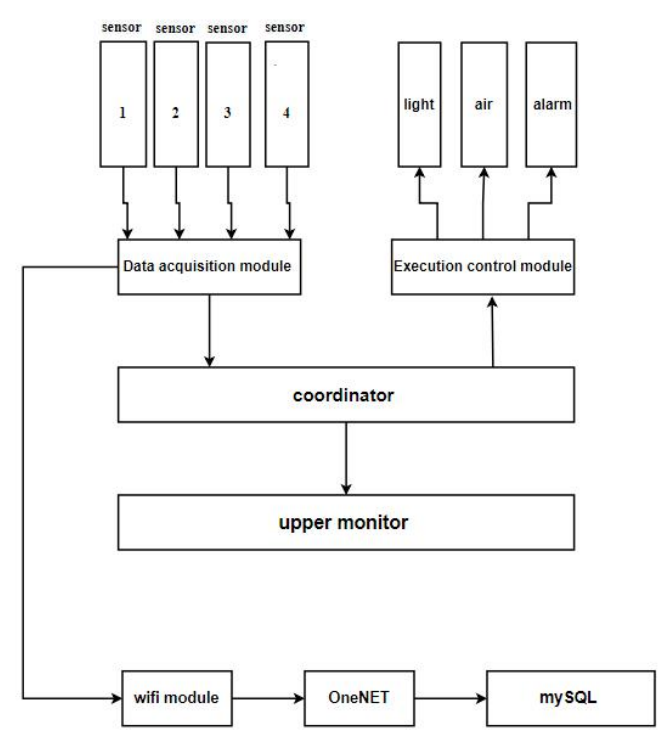

Fig.1. Technical route of the monitoring system

\subsection{Hardware design}


In this system, ZigBee components and corresponding terminals are mainly applied to collect the patchouli growth environment. The ZigBee motherboard integrates CC2530 MCs and ESP8266 WIFI modules.

(a) CC2530. cc2530 combines superior performance from the leading RF transceivers [6]. It has different operating modes, which makes it particularly adapted to systems with ultra-low power consumption requirements. Its short conversion time between operating modes further ensures low energy consumption. Its general structure is shown in Figure 2,3.

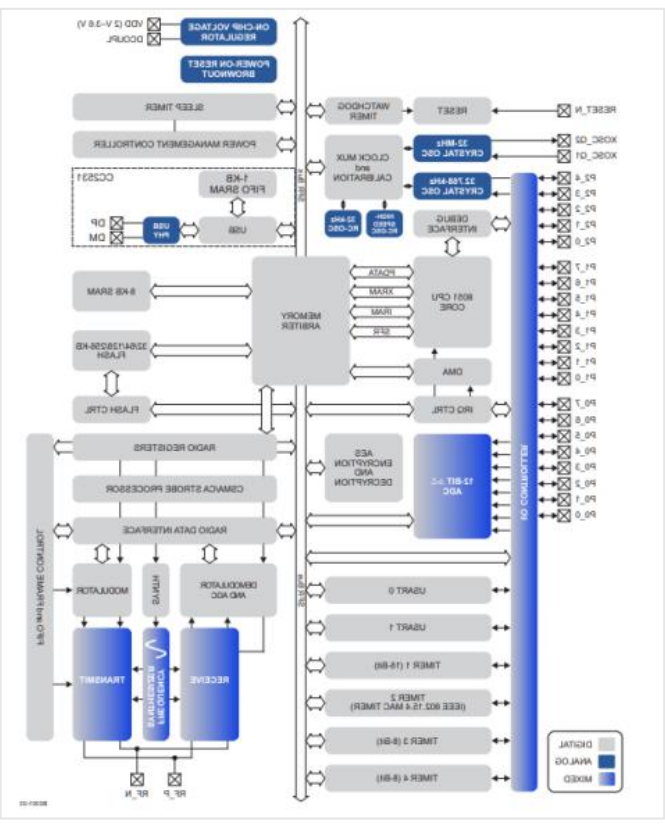

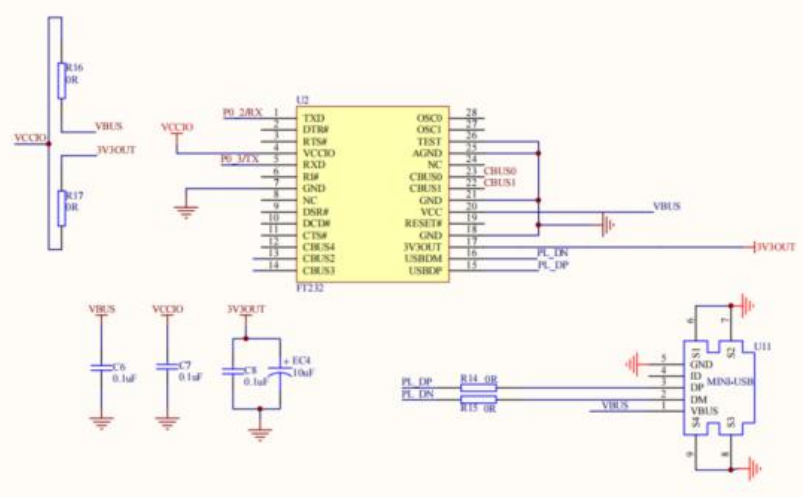

Fig.3. CC2530 Circuit Design(part)

(b) ESP8266. It integrates antenna switches, RF balun, power amplifier, low noise amplifier, filters, and power management modules [8]. This compact design requires minimal external circuits and minimize PCB size. Its internal structure is shown in Figure 4.

c. Related sensors. The sensors required for this design include MQ2 gas sensors, DHT11 temperature and humidity sensors, and infrared sensors. The sensors are shown in Figure 5.
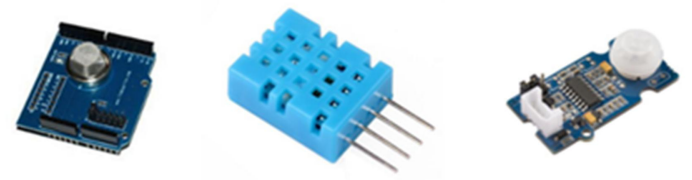

Fig.5. MQ2 gas sensors, DHT11 temperature and humidity sensors.

Fig.2. CC2530 diagrammatic sketch

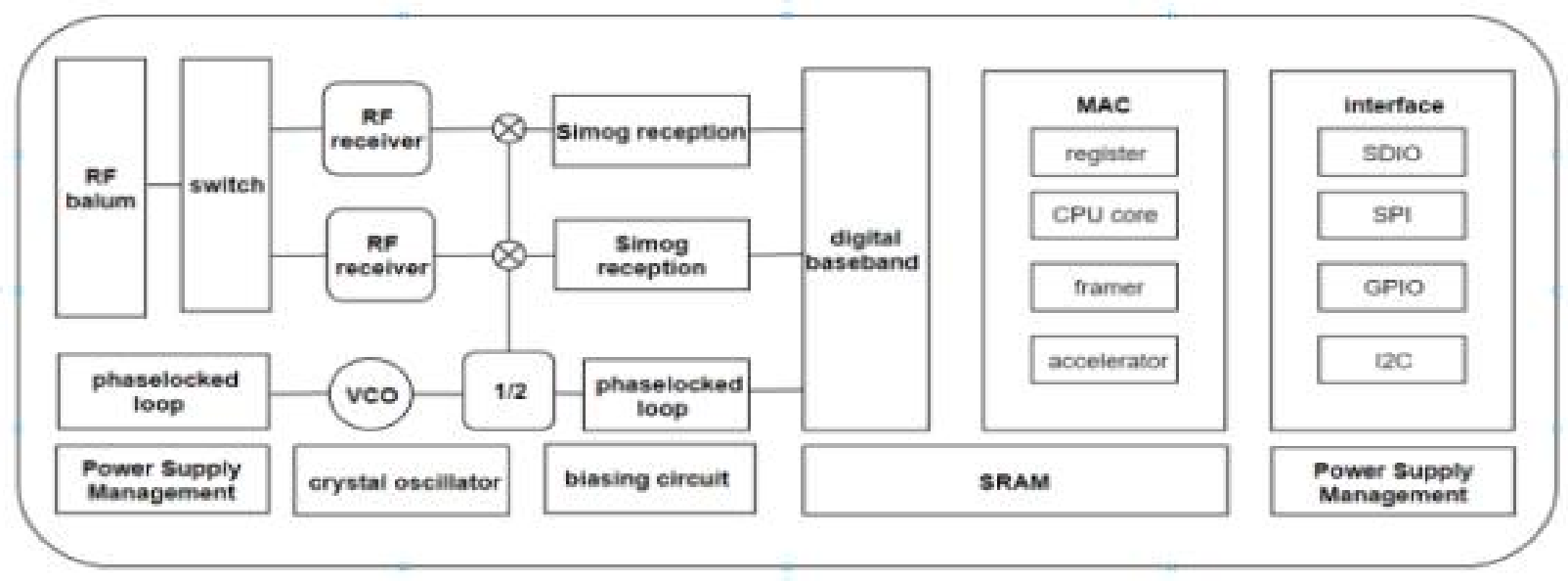

Fig.4. esp8266 struct diagram

\subsection{Software Design}

Software design is mainly the design of upper computer software. We connect the sensor to the ZigBee core control module and deploy it in the actual patchouli planting scenario; the pins of each sensor correspond one to one to the main board. The data collected by the sensor is transmitted through the coordinator. The WIFI module is 
used for wireless transmission between the coordinator and the terminal. And between the coordinator and the upper machine is connected by WIFI or serial port. The design work of the upper position machine mainly includes collecting the sensor data and displaying it in real time, judging whether the patchouli growth environment index according to the collected information is normal, and regulating the terminal equipment.

\subsection{OneNET Cloud Platform}

The OneNET cloud platform is an open platform for the PaaS IoT, and it is an efficient, stable and safe application platform for the IoT application between real devices [9]. From a device-oriented perspective, it can adapt to various network environments and common transmission protocols, and provide fast access schemes and device management services of various hardware terminals. From an application - oriented perspective, it provides rich API and data to meet the development needs of all industries of application systems. In short, the platform can help developers easily realize device access and equipment connection, quickly complete product development and deployment, and provide perfect Internet of Things solutions for intelligent hardware and smart home products.

Web server is able to obtain environmental parameters such as temperature and humidity collected by sensors from the patchouli planting scene from the OneNET cloud platform, and save these parameters as data types for java. The data transmission between the two also follows the HTTP protocol. Web server can access data from the cloud platform within seconds and save it into the mySQL database. We collect historical data and then use it as a sample studying the growth status of patchouli.

\section{System communication protocol}

\subsection{TCP/IP protocol}

Some IoT devices are initially designed to be compatible with TCP/IP protocols, thus enabling direct access to the Internet of Things, such as WIFI modules. TCP is a connection-oriented communication that, due to its characteristics, normal communication occurs only after the client is connected to the service side [10]. The protocol response mechanism and false packet retransmission mechanism provide TCP transmission with high transmission accuracy. The client connection to the service side is implemented in the form of a IP address plus port. Transmission using the TCP protocol reduces reduced speed and efficiency.

\subsection{HTTP protocol}

The full name of HTTP is the HyperText Transfer Protocol, proposed to provide and receive the HTML interface through which it transmits web's interface information over the Internet [11]. In IoT devices, some devices are not directly connected to the IoT, but need some protocols. For example, in embedded Web development, the web server to the hardware device.

\subsection{The MQTT protocol}

Message Queuing Telemetry Transport (MQTT) was an instant messaging protocol developed by IBM for the communication of remote sensors and control devices with limited mass computing capacity and working on lowbandwidth, unreliable networks [12]. The protocol uses the publishing/subscription message mode, while using TCP/IP to establish a network connection.

\section{Conclusion}

The contradiction between the production mode of medicinal plants and the protection and development of wild seed sources is growing. In especial, the expanding demand for commercial interests has put great pressure on the protection of wild medicinal plant resources. Therefore, through promoting the systematic and automatic planting of medicinal field management is of great significance to expand the scale of medicinal field planting, improve the growth quality of medicinal plants and solve the conflicts between artificial planting and wild medicinal plant resources.

We need to strengthen the artificial cultivation of patchouli and create higher productive cultivation techniques according to the defects shown in the current cultivation process. Meanwhile, due to pure artificial Patchouli planting, its cost is high, while automatic or semi-automatic planting 
management can effectively solve the problem of labor cost and be beneficial to the expansion of the planting scale.

\section{Acknowledgement}

This research was funded by the Special Project of Education Department of Shaanxi Provincial Government of china, grant number $16 \mathrm{JK} 1048$.

\section{References}

[1] Yueyang Li, Railway Su, Yin Wang, Xiaoping Ren, Hui Han, Tianzhu Zhang. Comparative analysis of facility gardening in China and the Netherlands $[\mathrm{J}]$. Chinese vegetables,2020(06):11-15.

[2] Yongde Guo. Israel International Water Technology and Environmental Control Exhibition Investigation Report [ J]. Science for Technology Forum,2008(07):43-45.

[3] Gongde He. Japanese Agriculture to Automation[N]. Scientific Guide,2005/03/16(A03).

[4] CaiYun Zhang et al. Comparative Analyses of the Chloroplast Genomes of Patchouli Plants and Their Relatives in Pogostemon (Lamiaceae)[J]. Plants, 2020, 9(11): 14971497.

[5] Zengzhe Lai, Huatong Zhang, Xujun He, Gang Wu, Jing Zhao. Planting and Management Technology of Patchouli Cuttings[J]. New Rural technology,2020(04):8-10.

[6] Xue Luo, Min Chen, Chun Zhu, Zhenzhen Ding. Research on Wireless temperature and humidity data acquisition and storage module based on ZigBee and CC2530[J]. Technological innovation and productivity,2021(04):66$68+71$.

[7] Guang Chen. Design of Data Acquisition Terminal Based on ZigBee Technology [J]. Digital Technology and Its Application, 2021, 39 (03): 132 - 134.

[8] Jinbo Qiu, Xuhua Qiu, Qiujing Zhang, Jiehua Wu. Design of Home Equipment Control System Based on the Three - layer Architecture of the Internet of Things [J]. Scientific and Technology Innovation, 2021 (13): 168 - 169.

[9] Lei Deng, Chengzhong Deng. Cloud Service - Based Remote Control System[J]. Electronic production, 2021 (09): 38 40
[10] Xiyi Nie. The Airport Runway Monitoring System based on the TCP/IP protocol $[\mathrm{J}]$. Electronic measurement technology abroad, 2021, 40 (02): 139 - 143.

[11] Jingfa Li, Weixin You, Ping Yi. Design of distributed topic map monitoring service based on HTTP protocol[J]. Changjiang Information and Communications, 2021, 34 (03): $118-121$.

[12] Chuyue Kong, Yu Chen, Qianming Zhao. Research on cloud side communication mapping of power distribution Internet of Things based on MQTT protocol[J]. Protection and Control of Power Systems, 2021, 49 (08): 168 - 176.

\section{Biographies}

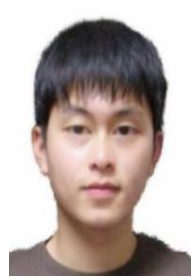

Guodong Li is studing in biology and medicine (medical big data direction) and is studying for a master's degree. During the research study, he participated in the IoT project, and the main direction is smart agriculture.

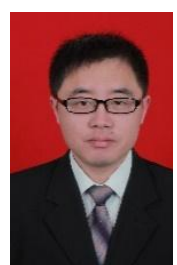

Ping Zhang Graduated with M.S at the Department of Computer and Information Science of Liaoning Normal University from 2008 to 2011 . He is a lecturer in Baoji University of Arts and Sciences. His current research interests include bioinformatics machine learning and graph neural network. 